\title{
Floater Dynamics Can Explain Positive Patterns of Density-Dependent Fecundity in Animal Populations
}

\author{
Vincenzo Penteriani, ${ }^{1, *}$ Fermín Otalora, ${ }^{2, \dagger}$ and Miguel Ferrer ${ }^{3, \ddagger}$
}

1. Department of Conservation Biology, Estación Biológica de Doñana, Consejo Superior de Investigaciones Científicas, Avenida María Luisa s/n, Pabellón del Perú, 41013 Seville, Spain;

2. Laboratorio de Estudios Cristalográficos, Instituto Andaluz de Ciencias de la Tierra (Consejo Superior de Investigaciones

Científicas/University of Granada), Campus Fuentenueva, Facultad de Ciencias, 18002 Granada, Spain;

3. Department of Biodiversity Conservation, Estación Biológica de Doñana, Consejo Superior de Investigaciones Científicas, Avenida María Luisa s/n, Pabellón del Perú, 41013 Seville, Spain

abstract: After some 70 years of debate on density-dependent regulation of animal populations, there is still poor understanding of where spatial and temporal density dependence occurs. Clearly defining the portion of the population that shapes density-dependent patterns may help to solve some of the ambiguities that encircle density dependence and its patterns. In fact, individuals of the same species and population can show different dynamics and behaviors depending on their locations (e.g., breeding vs. dispersal areas). Considering this form of intrapopulation heterogeneity may improve our understanding of density dependence and population dynamics in general. We present the results of individual-based simulations on a metapopulation of the Spanish imperial eagle Aquila adalberti. Our results suggest that high rates of floater mortality within settlement areas can determine a shift in the classical relationship (from negative to positive) between the fecundity (i.e., fledglings per pair) and density (i.e., number of pairs) of the breeding population. Finally, we proved that different initial conditions affecting the breeder portion of the population can lead to the same values of fecundity. Our results can represent a starting point for new and more complex approaches studying the regulation of animal populations, where the

\footnotetext{
* Corresponding author; e-mail: penteriani@ebd.csic.es.

+ E-mail: otalora@ugr.es.

† E-mail: mferrer@ebd.csic.es.
}

forgotten and invisible component-the floater-is taken into account.

Keywords: breeding performance, density-dependent fecundity, floater mortality, settlement areas.

Density dependence affects life-history traits and is one of the key processes in bird population dynamics; thus, understanding it is crucial in conservation biology (Lack 1954). In stable, saturated populations living in homogeneous habitats, density-dependent reproduction (i.e., the input rate of a population) generally shows a negative relationship with population density; that is, the higher the density, the lower the fecundity (e.g., Ferrer and Donázar 1996; Both 1998; Newton 1998; McCallum et al. 2000; Hixon et al. 2002; Paradis et al. 2002). Finally, there are many causes of density dependence (Dhondt et al. 1992; Both 1998; Ricklefs 2000; Hixon et al. 2002), and these can act simultaneously at different spatial scales (Rodenhouse et al. 2003).

A crucial link exists between the population dynamics of birds in their settlement (i.e., temporary settling zones used during dispersal by nonbreeders and in which they do not display territorial behavior) and breeding areas. Factors affecting the survival of floaters (i.e., dispersing individuals able to enter as breeders into the reproductive population when a breeding territory or a potential matethe owner of a suitable breeding territory-becomes available) also influence the dynamics of the whole population due to their effects on the breeding segment of the population in reproductive areas (Penteriani et al. 2005b). Furthermore, an increase in floater mortality within settlement areas can explain puzzling declines or extinctions of breeding populations (Penteriani et al. 2005a). In fact, we previously showed that (1) the number of floaters in a population is a function of the fecundity in the breeding territories (i.e., the higher the fecundity, the sooner an equilibrium is attained) and is limited by the availability of settlement areas, (2) environmental stochasticity has a lower impact on breeders when it takes place in a tem- 
porally and spatially disjunct way (in either settlement or breeding areas) and therefore affects only a portion of a population, and (3) an increase in floater mortality within settlement areas negatively affects the breeding population, reducing both the mean number of pairs and mean fecundity.

Such a strong relationship between floaters and breeders of the same population suggests that floater dynamics within settlement areas may affect breeding population properties such as density dependence. However, studies on density dependence have been focused mainly on the breeding portion of animal populations and consequently have been generally inattentive to the possible influences of what occurs to floaters. Because of the link between the dynamics in settlement and breeding areas (e.g., factors affecting floater survival also influence whole population dynamics), disperser mortality has the potential to be a crucial ecological mechanism by which density can affect demographic rates. Consequently, population studies that ignore floater dynamics (generally due to a lack of information on the location of settlement areas and the dynamics of individuals within them) may fail to understand all of the different factors that influence density-dependent population patterns. In this context, we agree with Turchin's (2003) view of density dependence, according to which such a property of an animal population must be considered as "a theoretical framework with which to investigate the causal factors of population fluctuations" and that "although quantifying density dependence does not uniquely identify the biological mechanisms responsible for various aspects of population dynamics, it allows us to make the first step toward this goal.” For example, there is a poor understanding of where both spatial and temporal density dependence occur (Sinclair 1989; Dhondt et al. 1992). Clearly defining the portion of the population (breeders and/or floaters) that shapes density-dependent patterns may help to solve some of the ambiguities that, after some 70 years of debate (Nicholson 1933), still surround density dependence and population dynamics in general (Murray 1994). In fact, intrapopulation heterogeneity represents an issue that was largely ignored in the context of density dependence but was shown to have important consequences (see Grimm and Uchmanski 2002, although in a different context). How do floaters alter density-dependent patterns within populations? To test this question we developed individual-based simulation models to explore the range of possible variations of density-dependent fecundity when individual dynamics within settlement areas are taken into account.

\section{Methods}

We based our work on a century-long time series of a metapopulation of the Spanish imperial eagle Aquila adalberti, which, with fewer than 150 pairs, is the most endangered bird of prey in Europe and one of the most threatened raptors in the world (Ferrer 2001). Using the same procedures as Penteriani et al. (2005a, 2005b), we analyzed the effects of floater mortality (ranging from $5 \%$ to $30 \%$ ) on the mean fecundity (i.e., the mean number of fledglings per year, averaged over all pairs in the population) of a breeding population under two different scenarios: (1) when the gradient of the age at first reproduction varied from 3 to 5 years and (2) for saturation levels of 10,15, and 20 breeding pairs. We explored these ranges of values for age at first reproduction and saturation levels of the population because they best reflect the real scenarios that we observed for the Spanish imperial eagle in Doñana (southwestern Spain). The values of floater mortality reflects the real range of possible values that we recorded in the field (e.g., Ferrer et al. [2004] reported an annual mortality of 0.26 】 0.30 for unpaired eagles).

\section{Empirical and Theoretical Basis of Simulations}

The Spanish imperial eagle population of Doñana consists of a maximum of 16 pairs. This species is a large $(2,500-$ $3,500 \mathrm{~g})$, sedentary, and territorial bird of prey that is characterized by low reproductive rates (averaging 0.75 chicks per pair per year), a density-dependent immature phase of 3-5 years (most frequently five), and a maximum longevity of 22 years. Typically, breeding pairs include two adults, but some pairs may be mixed; that is, one or both mates have not attained adult plumage (!5 years old). Thirty floaters (60\% of the whole disperser population) were marked with radio tags over approximately 15 years (Ferrer 2001). Because data collection started in 1890, our long-term data set represents a strong basis for the general understanding of the dynamics of a small population and the building of realistic, predictive models accounting for the general relationship (e.g., breeding performance) between floaters and breeders of a population of a predator species. The structure, parameters, and initial values of our models were guided and set by the observed longterm (more than one century) eagle population dynamics, for which previous simulations proved the similarity between modeled patterns and empirical data (Ferrer and Penteriani 2003; Ferrer et al. 2004; Penteriani et al. 2006).

Because in previous analyses (Penteriani et al. 2005a, $2005 b$ ) we highlighted that environmental stochasticity has a stronger influence on the whole population when variations in environmental conditions occur in a synchronous way (i.e., in both the settlement and breeding areas at the same time), the simulations were performed under the less adverse scenario, that is, when the variations in environmental conditions occur asynchronously. An increase in floater mortality could be due to environmental 
stochasticity (e.g., natural catastrophes and habitat destruction or fragmentation) or human-induced deaths (e.g., poisoning, electrocution).

Our model included density-dependent compensation because models without density-dependent compensation inevitably lead to the demise or explosion of a population as well as erroneous inferences about a population's responses to individual intra- and interspecific interactions (Emlen et al. 2003). All the simulations start at time $t$ P 0 with 15 eagle pairs and 15 floaters (to simulate real situations). We ran 100 simulations (100 years for each of them), and at the end of each series of 100 simulations, the model generated the mean of number of pairs and fecundity (fledged young) for the 100-year period for each simulation. See the appendix in the online edition of the American Naturalist for the full description of the algorithms and parameters used in the simulation.

\section{Output Analyses}

Spearman tests explored the direction of the relationship between mean fecundity and population density (i.e., number of pairs) under different conditions of annual floater mortality. Mann-Whitney $U$-tests compared the patterns in fecundity for several different combinations of ages at first reproduction and saturation thresholds, when mortality in settlement areas varied from 5\% to 30\%. All means are given with $\mathbf{1} \mathrm{SD}$, all tests are two-tailed, and statistical significance was set at $P$ ! .05. The software package used was SPSS, version 10.0.

\section{Results and Discussion}

The patterns shown by the density-dependent fecundity under different rates of floater mortality highlighted the occurrence of three phenomena within the breeding population. First, we observed an inversion in the direction of the density dependence of fecundity, that is, from negative to positive density dependence (Sinclair 1989), when floater mortality increased (fig. 1) and consequently depressed the number of breeders. That is, extremely high mortalities of floaters in the settlement areas are able to engender (1) a decrease in the number of breeders due to the lack of new mates available to replace the lost ones (i.e., increasing difficulty of pair formation) and, consequently, (2) a positive density-fecundity relationship in the breeding portion of the population. A possible scenario accounting for a positive relationship between breeding pair density and fecundity (i.e., the Allee effect; Allee et al. 1949) could be the one in which breeder density decreases well below the saturation density of the population due to high levels of floater mortality (see Courchamp et al. 1999 for a review of scenarios and factors involved in generating inverse density dependence at low densities). This would imply that when floater mortality increases, a corresponding decrease of the floater availability as potential mates does not allow us to replace all the vacancies in the breeding population. The difficulty in encountering mates at low densities is a scenario typical of the Allee effect, determining a disproportionate reduction in fecundity at small population sizes (Amarasekare 1998). Such a correspondence between the outputs of our simulation and previous information on population dynamics (e.g. Lande 1987; Dennis 1989; Amarasekare 1998, Lande et al. 2003) indicates that our explanation might be correct, but a more thorough future analysis is needed to fully understand positive density dependence fecundity in animal (meta)populations.

The thresholds of mortality rate at which mean fecundity shifted from a negative to a positive relationship with number of pairs mainly corresponded to $25 \%$ and $30 \%$. In fact, (1) for all ages at first reproduction and saturation thresholds of the breeding population, the pattern of density-dependent fecundity was always positive (significance levels equal to $P$ ! .0001; Spearman tests) when the floater mortality was $30 \%$, and (2) when the floater mortality was $25 \%$, fecundity showed a positive pattern for ages of 4 and 5 years and thresholds of 10 pairs (significance levels ranging from $P$ P .002 to $P$ ! .0001; Spearman tests). It is interesting to highlight that, as predicted for the factors affecting population changes (Turchin 1999), floater mortality showed the properties of densitydependent endogenous factors, that is, dynamic feedbacks affecting population numbers and involving time lags. In fact, the effects of floater mortality appear in the breeding areas only after several decades (Penteriani et al. 2005a). Although negative density-dependent fecundity has been previously described for our eagle population (Ferrer and Donázar 1996), some periods of positive relationship between fecundity and density have been also detected (M. Ferrer and V. Penteriani, unpublished data). Taking floater dynamics within settlement areas into consideration can explain otherwise inexplicable positive density-dependent patterns in breeding populations.

Second, the fecundity of our population showed similar values for different rates of floater mortality (i.e., different pressures acting on the population) for both the age at first reproduction (fig. 1A) and saturation thresholds (fig. $1 B$ ). This means that the same values of fecundity that we observe in a population can be the results of (at least) two different, spatially distinct factors acting on the whole population, that is, (1) the classical density-dependent effect due to population saturation or (2) the effect of high mortalities in the floater sector of the population. Consequently, different initial conditions (i.e., a stable and saturated population or high rates of floater mortality 


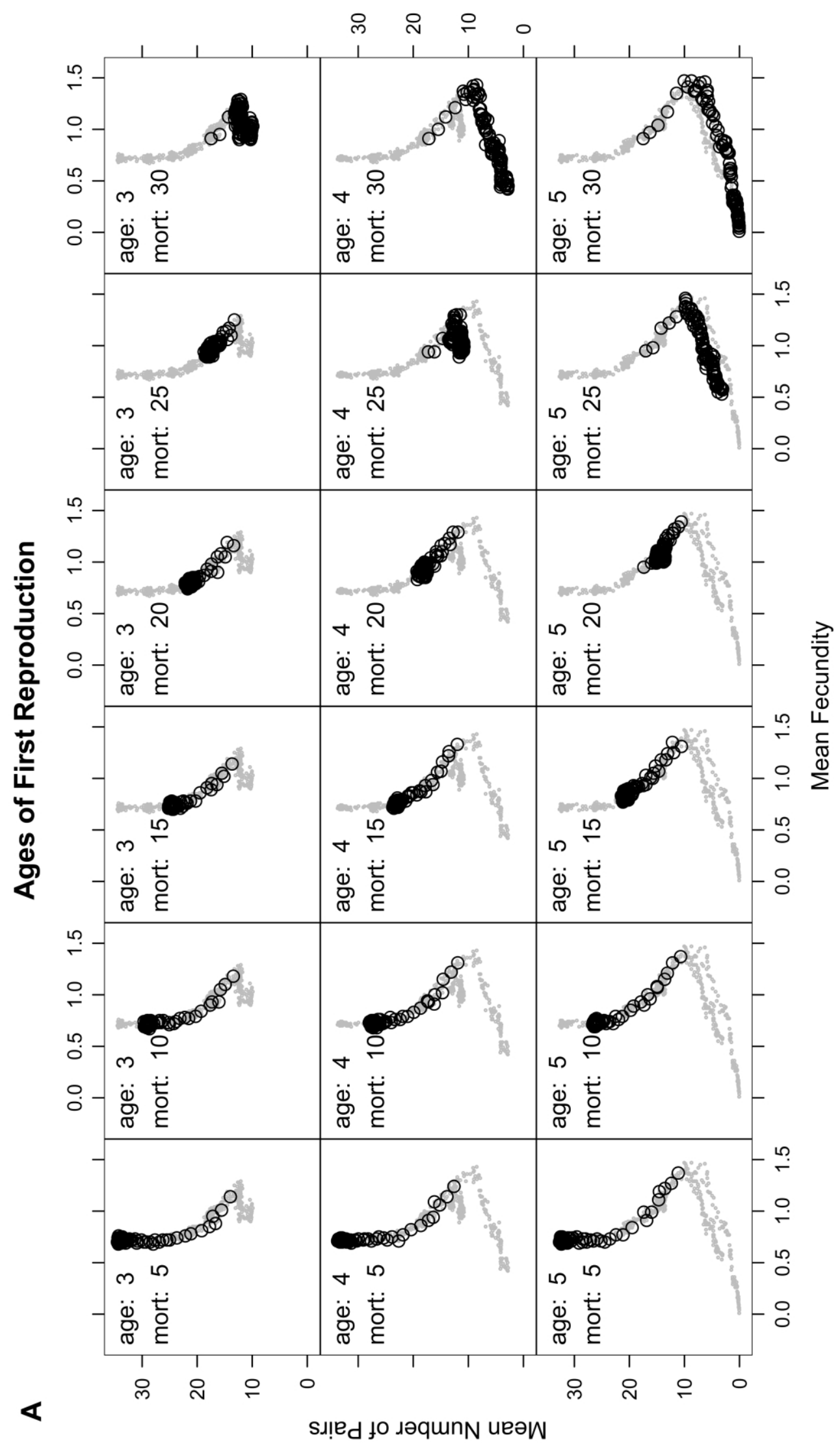




\section{Population Capacity}

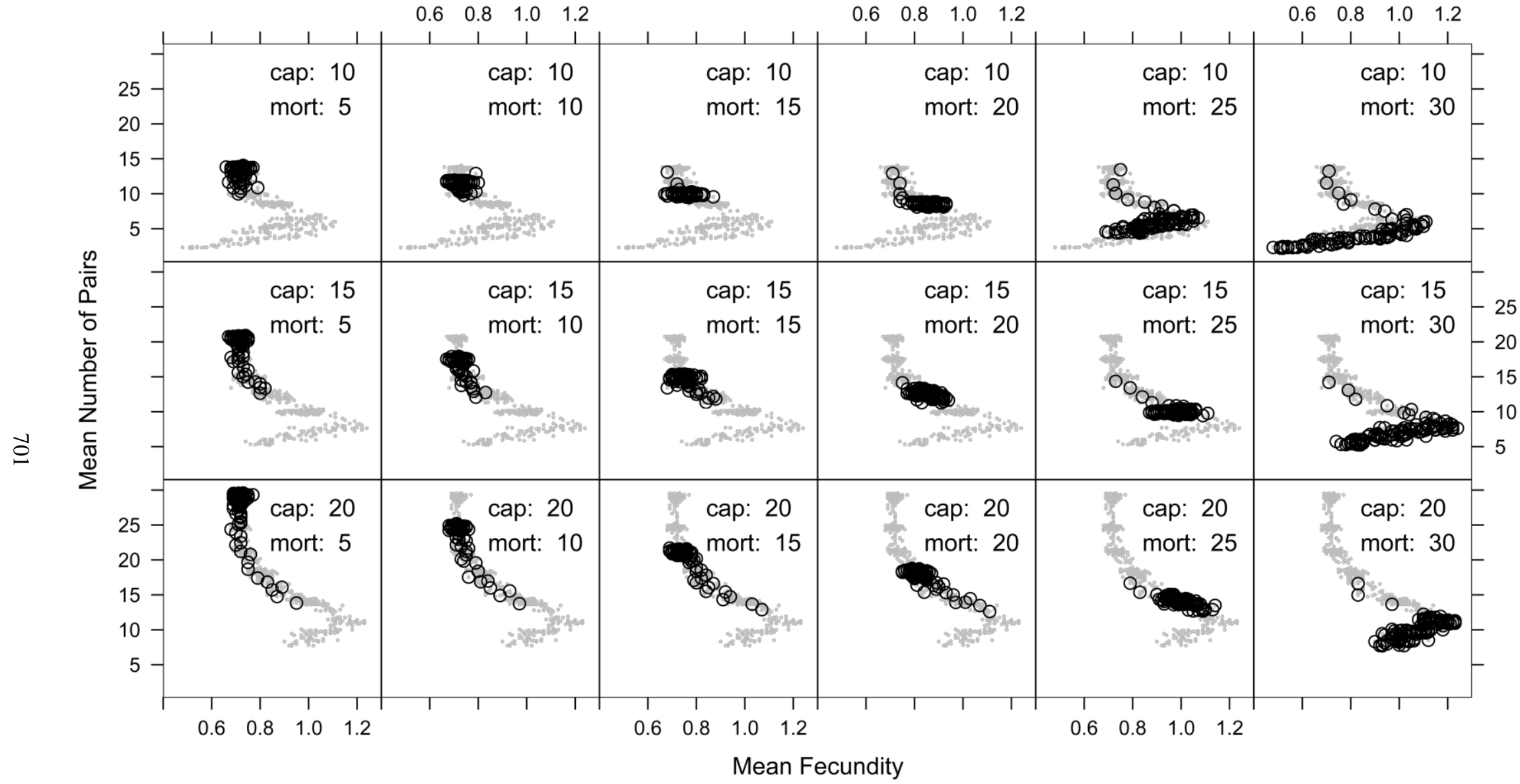

Figure 1: Patterns of density-dependent fecundity when floater mortality (mort) shifts from $5 \%$ to $30 \%$. Gray trajectories represent the whole range shown by fecundity when floater mortality has been varied from $5 \%$ to $30 \%$, whereas black circles show the specific fecundity for each level of floater mortality for both ages at first reproduction (age in A) and population capacity (cap in $B$ ). Bird populations can shift from negative to positive density-dependent fecundity as a result of increasing mortality rates of floaters within settlement areas. Similar patterns in breeding performance arise when simulating both different ages at first reproduction (from 3 to 5 years old; $A$ ) and population thresholds (from 20 to 10 pairs; $B$ ). That is, different departure conditions of the breeding populations can determine similar breeding performance. In fact, the same values of mean fecundity can appear in the same breeding population under extremely different situations (e.g., saturated and stable population vs. increasing or unstable population), depending on the levels of floater mortality within settlement areas. 
determining a decline in the population) produced similar effects (i.e., the same values of mean fecundity) in the breeding sector of the population. This means we could potentially be confronted with inferential fallacy (Alker 1969), quite relevant but often probably overlooked by analyses on population dynamics and regulation when individual processes are deduced from wide patterns. In fact, mistakes may arise by inferring processes or patterns at one level of analysis (e.g., density dependence in the breeding population) from those occurring at another level (e.g., floater mortality within settlement areas).

Third, both earlier ages at first reproduction and higher saturation thresholds in the breeder section of the population buffered the negative effect of increasing floater mortality (see also Ferrer et al. 2004). In fact, the lowest values of mean fecundity (lowest tails in fig. 1) mainly characterized higher ages at first reproduction and lower saturation thresholds. That is, both a lower age at first reproduction and a higher population threshold were able to reduce the effect of floater mortality in the breeding sectors. Once more, the pressure of two independent and different factors (i.e., younger age of first reproduction and higher saturation levels or higher age of first reproduction and lower saturation levels) generated similar patterns. These pairs of similar patterns were (see fig. 1) 3 years old and 20 breeding pairs, 4 years old and 15 breeding pairs, and 5 years old and 10 breeding pairs. In particular, (1) breeding performances of populations of 3-year-olds at first reproduction were similar to breeding performances of populations with a saturation threshold fixed at 20 pairs when floater mortality was $5 \%(z \mathbf{P}-0.65, P \mathbf{P} .52 ; n \mathbf{P} 100,100$; Mann-Whitney $U$-test), $15 \%$ (z $\mathbf{P}-0.79, P \mathbf{P} .43$ ), and 30\% (z P - 0.03, P P .98); (2) populations of 5 years old at first breeding reproduced similarly to those with a threshold of 10 pairs when floater mortality was 5\% (z P -1.27, P $\mathbf{P}$.20) and 25\% ( z P -1.34, P P .18); and (3) all three age combination thresholds showed similar breeding performances (all $z$ from -1.80 to -0.86 and all $P$ from 0.39 to 0.07 ) when floater mortality was $10 \%$. To conclude, the buffer mechanisms that were shown to act on our small population support the importance of taking into consideration buffers in population analyses, as previously highlighted by Ferrer et al. (2004) and Grimm et al. (2005). In fact, buffer mechanisms are able to reduce the environmental noise (i.e., the variation of a population's growth rates due to environmental variations; Burgman et al. 1993), a key determinant of the extinction risk of small populations (Wissel et al. 1994).

In the same way that population dynamics within breeding areas have a strong influence on dispersal (Murrell et al. 2002), it is crucial to consider floater dynamics when modeling population dynamics for the whole population (breeders + dispersers). The study of population dynamics in dispersal areas warrants the same levels of interest and awareness as breeding population studies; the result can be answers to population regulation, changes, alterations, or simply demographic tendency in breeding territories.

\section{Acknowledgments}

This note has been greatly improved by comments from J. Bascompte, M. M. Delgado, C. Melián, M. Wichmann, and an anonymous reviewer. During this research, V.P. was supported by a contract from the program Incorporación de Investigadores al Sistema Español de Ciencia y Tecnología (CCAA de Andalucía) and a Marie Curie Fellowship from the European Commission program Improving Human Research Potential and the Socio-Economic Knowledge Base, under contract number HPMF-CT-200001098. The author is solely responsible for the information communicated, and the European Commission is not responsible for any view or result expressed.

\section{Literature Cited}

Alker, H. R., Jr. 1969. A typology of ecological fallacies. Pages 6986 in M. Dogan and S. Rokkan, eds. Quantitative ecological analysis in the social sciences. MIT Press, Cambridge, MA.

Allee, W. C., A. E. Emerson, O. Park, and K. P. Schmidt. 1949. Principles of animal ecology. Saunders, Philadelphia.

Amarasekare, P. 1998. Allee effects in metapopulation dynamics. American Naturalist 152:298-302.

Both, C. 1998. Density dependence of clutch size: habitat heterogeneity or individual adjustment? Journal of Animal Ecology 67: 659-666.

Burgman, M. A., S. Ferson, and H. R. Akcakaya. 1993. Risk assessment in conservation biology. Chapman \& Hall, London.

Courchamp, F., T. Clutton-Brock, and B. Grenfell. 1999. Inverse density dependence and the Allee effect. Trends in Ecology \& Evolution 14:405-410.

Dennis, B. 1989. Allee effects: population growth, critical density, and the chance of extinction. Natural Resource Modeling 3:481-538.

Dhondt, A. A., B. Kempenaers, and F. Adriaensen. 1992. Densitydependent clutch size caused by habitat heterogeneity. Journal of Animal Ecology 61:643-648.

Emlen, J. M., D. C. Freeman, M. D. Kirchhoff, C. L. Alados, J. Escos, and J. J. Duda. 2003. Fitting population models from field data. Ecological Modelling 162:119-143.

Ferrer, M. 2001. The Spanish imperial eagle. Lynx, Madrid.

Ferrer, M., and J. A. Donázar. 1996. Density-dependent fecundity by habitat heterogeneity in an increasing population of Spanish imperial eagles. Ecology 77:69-74.

Ferrer, M., and V. Penteriani. 2003. A process of pair formation leading to assortative mating: passive age-assortative mating by habitat heterogeneity. Animal Behaviour 66:137-143.

Ferrer, M., F. Otalora, and M. García-Ruiz. 2004. Density-dependent age of first reproduction as a buffer affecting persistence of small populations. Ecological Applications 14:616-624.

Grimm, V., and J. Uchmanski. 2002. Individual variability and population regulation: a model of the significance of within-generation density dependence. Oecologia (Berlin) 131:196-202. 
Grimm, V., E. Revilla, J. Groeneveld, S. Kramer-Schadt, M. Schwager, J. Tews, M. C. Wichmann, and F. Jeltsch. 2005. Importance of buffer mechanisms for population viability analysis. Conservation Biology 19:578-580.

Hixon, M. A., S. W. Pacala, and S. A. Sandin. 2002. Population regulation: historical context and contemporary challenges of open vs. closed systems. Ecology 83:1490-1508.

Lack, D. 1954. The natural regulation of animal numbers. Oxford University Press, Oxford.

Lande, R. 1987. Extinction thresholds in demographic models of territorial populations. American Naturalist 130:624-635.

Lande, R., S. Engen, and B.-E. Sæther, 2003. Stochastic population dynamics in ecology and conservation. Oxford University Press, Oxford.

McCallum, H., J. Kikkawa, and C. Catterall. 2000. Density dependence in an island population of silvereyes. Ecology Letters 3:95100 .

Murray, B. G. 1994. On density dependence. Oikos 69:520-523.

Murrell, D. J., J. M. J. Travis, and C. Dytham. 2002. The evolution of dispersal distance in spatially-structured populations. Oikos 97: 229-236.

Newton, I. 1998. Population limitation in birds. Academic Press, San Diego, CA.

Nicholson, A. J. 1933. The balance of animal populations. Journal of Animal Ecology 2:132-178.

Paradis, E., S. R. Baillie, W. J. Sutherland, and R. D. Gregory. 2002. Exploring density-dependent relationships in demographic parameters in population of birds at a large spatial scale. Oikos 97:293307.

Penteriani, V., F. Otalora, F. Sergio, and M. Ferrer. 2005a. Environ- mental stochasticity in dispersal areas can explain the "mysterious" disappearance of breeding populations. Proceedings of the Royal Society B: Biological Sciences 272:1265-1269, doi: 10.1098/ rspb.2005.3075.

Penteriani, V., F. Otalora, and M. Ferrer. 2005b. Floater survival affects population persistence: the role of prey availability and environmental stochasticity. Oikos 108:523-534.

Penteriani, V., M. A. Fortuna, C. J. Melián, F. Otalora, and M. Ferrer. 2006. Can prey behaviour induce spatially synchronic aggregation of solitary predators? Oikos 113:497-505.

Ricklefs, R. E. 2000. Density dependence, evolutionary optimisation, and the diversification of avian life histories. Condor 102:9-22.

Rodenhouse, N. L., T. S. Sillett, P. J. Doran, and R. T. Holmes. 2003. Multiple density-dependence mechanisms regulate a migratory bird population during the breeding season. Proceedings of the Royal Society B: Biological Sciences 270:2105-2110.

Sinclair, A. R. E. 1989. Population regulation in animals. Pages 197241 in J. M. Cherrett, ed. Ecological concepts. Blackwell Scientific, Oxford.

Turchin, P. 1999. Population regulation: a synthetic view. Oikos 84: 153-159.

. 2003. Complex population dynamics: a theoretical/empirical synthesis. Princeton University Press, Princeton, NJ.

Wissel, C., T. Stephan, and S.-H. Zaschke. 1994. Modelling extinction and survival of small populations. Pages $67-103$ in H. Remmert, ed. Minimum animal populations. Ecological studies. Vol. 106. Springer, Berlin.

Associate Editor: Volker Grimm Editor: Donald L. DeAngelis 